\title{
El empleo manufacturero en México, 1970-2013: un análisis espacial desde el enfoque de la NEK
}

\section{Manufacturing employment in Mexico, 1970-2013: a spatial analysis from the NKE approach}

\author{
Luis Brito Cruz* \\ Pablo Mejía Reyes**
}

\begin{abstract}
The evolution of manufacturing employment in Mexico between 1970 and 2013, was analyzed using a spatial data panel with the aim of identifying spatial interaction effects at the state level. Furthermore, under the New Keynesian Economy approach, it is assumed that the employment of this industry is positively correlated with total gross production and negatively with real wages and total factor productivity. Evidence was found of the effects of spillovers and spatial feedback rates indicating intraregional processes of circular and cumulative reinforcement that favor job creation.
\end{abstract}

Keywords: employment, manufacturing industry, production, space dependence, data panel.

\section{Resumen}

En esta investigación se analiza la evolución del empleo manufacturero en México entre 1970 y 2013, mediante panel de datos espaciales, con el objetivo de identificar efectos de interacción espacial a nivel de estados. Además, bajo el enfoque de la Nueva Economía Keynesiana se asume que el empleo de esa industria se correlaciona positivamente con la producción bruta total y, de manera negativa, con los salarios reales y la productividad total factorial. Se halló evidencia de efectos de desbordamientos y tasas de retroalimentación espacial que indican procesos intrarregionales de reforzamiento circular y acumulativo que favorecen la generación de empleo.

Palabras clave: empleo, industria manufacturera, producción, dependencia espacial, panel de datos.

* Facultad de Economía de la Universidad Autónoma del Estado de México, correo-e: luis_brito_cruz@hotmail.com

** Centro de Investigación en Ciencias Económicas, Facultad de Economía de la Universidad Autónoma del Estado de México, correo-e: pmejiare@uaemex.mx 


\section{Introducción}

Ante la globalización e internacionalización de la economía, la ubicación espacial de la producción ha sido un tema central en el análisis económico y la toma de decisiones. El cambio en el ritmo de crecimiento de las ciudades, la mayor movilidad de las personas y los productos especializados se han acentuado en regiones económicamente exitosas, como en Norteamérica, Europa occidental y Asia nororiental, que al facilitar la concentración de la producción también fomentaron la creación de empleos e instituido políticas orientadas a equilibrar espacialmente sus niveles de crecimiento y desarrollo. Sin embargo, otras regiones en desarrollo no terminan de integrarse a estas dinámicas territoriales, entre ellas América Latina, Asia suroriental y África subsahariana (World Bank, 2009).

Si bien actualmente no existe una teoría general que explique de manera consensuada los determinantes del crecimiento a nivel espacial, sí se han desarrollado enfoques y modelos que atribuyen la convergencia-divergencia regional a la dotación inicial de los factores de las áreas geográficas, el comercio intra e inter-regional y aspectos derivados del comportamiento de los agentes y la tecnología.

Además, esencialmente consideran que existe una estrecha relación entre la cantidad de bienes y servicios demandados y los costos de transporte que los consumidores deben cubrir para acceder a ellos; por lo que estos costos implícitamente inhiben o animan tanto a los flujos de consumidores como a las decisiones de localización de las empresas. ${ }^{1}$

De acuerdo con Sobrino (2016), la localización industrial se analiza como una relación persistente centro-periferia, con tendencia a la polarización territorial, donde influyen factores determinantes como la globalización, la nueva división internacional del trabajo, la flexibilización del proceso productivo, las crisis financieras y la desindustrialización.

La economía espacial analiza la forma en que los agentes toman decisiones de localización para concentrarse o dispersarse y permite comprender cómo es que la ubicación determina la capacidad de generación de puestos de trabajo en las unidades espaciales. Este es un aspecto relevante para prácticamente todos los países, dado que las altas tasas de desempleo registradas en distintos países y regiones del mundo provienen de la compleja

${ }^{1} \mathrm{Al}$ respecto, la teoría de interacción espacial (TIE) reconoce a los costos de transporte como el principal elemento que relativiza el valor de los lugares en función de su situación geográfica, pues éstos se relacionan negativamente con la intensidad de las interacciones entre las unidades espaciales; de esta manera, "el consumidor no asistirá a unidades que estén localizadas demasiado distantes en términos económicos (costos de transporte), temporales (tiempo de transporte), de inconvenientes (incomodidades del viaje) o energéticos (esfuerzo físico que requiere el viaje), por ejemplo" (Garrocho, 2012: 88). Además, según el tipo de unidades en que se midan, los costos de transporte pueden adoptar un carácter objetivo o subjetivo. 
diversidad económica que cada uno representa, así como de las interacciones espaciales al interior y entre ellos (OIT, 2019).

En este marco se explica porque la reestructuración económica experimentada en México durante la década de los ochenta provocó cambios significativos en los mercados de trabajo, que se acentuaron con la apertura comercial y la caída del PIB en 1995 (Pérez-Mendoza, 2005); asimismo, esta situación tuvo como consecuencia una reconfiguración diferenciada a nivel espacial y sectorial del empleo, así como la adopción de nuevas prácticas laborales tendientes a flexibilizar las formas de contratación e impulsar la productividad; por otra parte, las disparidades en los salarios y la productividad se ampliaron al dificultarse el crecimiento y desarrollo de los estados por los efectos de las crisis y recesiones, lo mismo que por la creciente migración, la inequitativa distribución del ingreso y la concentración de la fuerza de trabajo en actividades con bajo valor agregado.

Entre los sectores económicos, igualmente se remarcaron las diferencias en el grado de exposición a flujos comerciales y financieros internacionales, así como en la demanda de sus bienes o servicios, lo que condujo a discrepancias en sus tasas de crecimiento, dichos elementos propiciaron desequilibrios en el mercado de trabajo, que los estados han asimilado de manera heterogénea según su ubicación espacial, actividades predominantes, factores productivos disponibles y diversas características inherentes a ellos que los hacen más o menos resilientes. ${ }^{2}$

El objetivo general de esta investigación es analizar espacialmente al empleo en la industria manufacturera (POT) durante el periodo 1970$2013^{3}$ considerando los 32 estados mexicanos, así como cuantificar los efectos de interacción que sobre él generan la producción bruta total (PBT), la productividad total factorial (PTF) y los salarios reales (RM). Con base en la hipótesis de salarios de eficiencia postulada por la Nueva Economía Keynesiana (NEK), se asume que: i) PBT es el principal determinante del empleo con una relación directa, pues frente a un mayor nivel de actividad económica las empresas incrementan su demanda laboral; ii) se espera que RM tenga un efecto negativo debido a su rigidez y a que es uno de los mayores costos de la producción; iii) ante una mayor eficiencia marginal de los factores - particularmente del capital- se reduce la demanda de fuerza de trabajo, por lo que se considera que PTF también tiene un efecto negativo.

\footnotetext{
${ }^{2}$ Existe evidencia de que a largo plazo los procesos de divergencia entre las entidades del país prevalecen sobre los de convergencia (Asuad Sanén y Quintana Romero, 2010; Ocegueda Hernández 2015; Rodríguez Benavides et al., 2016).

${ }^{3}$ El análisis se limita a este periodo debido a que hasta marzo de 2020 los datos disponibles referentes a los Censos Económicos 2019 aún eran preliminares y no desagregados.
} 
Los argumentos de la Nueva Geografía Económica (NGE) son considerados para interpretar las posibles interacciones espaciales entre los estados mexicanos; el análisis econométrico se basa en el uso de modelos de datos de panel espacial, se toma como referencia la metodología "de lo general a lo particular", expuesta por Elhorst (2014).

Entre las contribuciones que esta investigación busca hacer se encuentra el análisis de los efectos que las distintas políticas económicas y estilos de crecimiento han tenido sobre el comportamiento del empleo en las entidades del país y en un horizonte temporal que permita identificar tendencias de largo plazo en la formación de aglomeraciones. Asimismo, al emplearse el enfoque teórico de la NEK es posible justificar a la PBT, RM y PTF como los principales determinantes del empleo manufacturero, particularmente, al observar que en México son escasos y cortos los periodos de crecimiento sostenido y que los salarios reales fueron poco flexibles porque nominalmente estuvieron sujetos a regulación (fijación de salarios mínimos).

Otro interés del estudio es cuantificar los diferentes efectos espaciales con el fin de inferir y entender los mecanismos bajo los cuales se incentiva o restringe la generación de empleo en la industria manufacturera, bajo el supuesto de reforzamiento circular y acumulativo.

Los principales hallazgos indican que se presentan efectos espaciales directos que corroboran las hipótesis asociadas al enfoque de los salarios de eficiencia; de la misma forma, se observaron contribuciones similares de los efectos directos e indirectos, lo que permite inferir que ocurren procesos homogéneos de diversa índole entre los estados que forman aglomeraciones. Lo más relevante es que se evidenciaron efectos de retroalimentación que deben aprovecharse y reproducirse en otras regiones, a fin de favorecer la creación de nuevos puestos de trabajo.

El resto del artículo consta de cinco apartados: primero se presentan las consideraciones teóricas de la NEK, enfatizándose la perspectiva de los salarios de eficiencia y resaltando la importancia de la dimensión espacial en los estudios económicos; luego se exponen los hechos estilizados y la evidencia empírica que encuadra la problemática de la escasa generación de empleo en la manufactura y su reconfiguración espaciotemporal; la tercera sección detalla el análisis exploratorio de los datos espaciales, el cálculo de la PTF y la metodología de los modelos de panel con componente espacial. La estimación y evaluación econométrica se presentan en el cuarto apartado, donde se discuten los resultados y se contrasta la evidencia empírica con los postulados teóricos considerados para el análisis. En las conclusiones se exponen los principales hallazgos. 


\section{El mercado de trabajo en la perspectiva de la NEK y la NGE}

La NEK asume que ante un salario que no vacía al mercado, los trabajadores no se sitúan sobre su curva de oferta y surge una situación de desempleo involuntario, en la que los individuos desean trabajar incluso por un sueldo inferior al vigente, pero no pueden hacerlo; también se basa en la relación convencional positiva entre los salarios reales y la oferta laboral -y negativa con relación a la demanda laboral-. También considera que debido a rigideces y fallas de coordinación entre las fuerzas del mercado, el nivel de empleo depende tanto de la demanda efectiva como de un salario real fijo superior al de equilibrio, denominado salario de eficiencia (Gordon, 1990).

De acuerdo con Mankiw y Romer (1991), las empresas tienen incentivos para pagar un salario superior al de equilibrio, aun cuando ello les genere mayores costos, esto atrae hacia ellas a los trabajadores más capacitados, estimula el sentimiento de lealtad y compromiso hacia la empresa y disminuye los costos de supervisión y monitoreo. Así, los salarios reales determinan los esfuerzos de los trabajadores y, por ende, su productividad.

Los salarios superiores a los de equilibrio pueden crear desempleo, pues el nivel efectivo de empleo se determina por la demanda laboral correspondiente al salario de eficiencia, lo cual genera una cantidad de empleo más baja que la de equilibrio del mercado. ${ }^{4}$ De esta manera, el salario se determina por un proceso de optimización que supone una economía con empresas idénticas y perfectamente competitivas con la siguiente función de producción:

$$
Y=A f[e(w) L], \operatorname{con} e^{\prime}(w)>0
$$

donde $Y$ es el producto de la empresa, $A$ representa un factor de cambio de la productividad, $e$ es el esfuerzo por trabajador, $w$ es el salario real y $L$ es el insumo de trabajo; entonces $e(w) L$ son las unidades de empleo efectivo. La condición $e^{\prime}(w)>0$ indica que el esfuerzo es una función creciente del salario real, por lo que se supone que el esfuerzo crece más que proporcionalmente para salarios bajos y lo contrario ocurre para salarios más elevados. ${ }^{5}$

\footnotetext{
${ }^{4}$ También es posible que un aumento en los salarios de eficiencia incremente la productividad, tal que la curva de demanda laboral se desplace hacia arriba expandiendo los requisitos laborales en cada nivel salarial. Esto implicaría una relación positiva entre ambas variables (Mejía-Reyes y TorresPreciado, 2020).

${ }^{5}$ Actualmente se discute el vínculo entre los salarios de eficiencia y el progreso tecnológico, pues los desajustes entre la demanda y oferta laboral se atribuyen a la sustitución de tareas algorítmicas o rutinarias por aquéllas de tipo cognitivas no rutinarias; también a fenómenos como la sobre calificación, la discriminación laboral, mayores diferencias salariales regionales y la tendencia de las grandes empresas a pagar salarios más altos no siempre respaldados por un incremento de la productividad laboral (Schlicht, 2016).
} 
Para analizar la determinación del empleo a partir del producto, los salarios y la productividad, como sus determinantes, se ha asociado de manera ad hoc el enfoque de los salarios de eficiencia con el modelo de crecimiento de Solow (1957). Se consideró el caso especial de cambio tecnológico neutral, en el que las tasas marginales de sustitución de los factores simplemente incrementan o disminuyen el producto alcanzable dado un nivel de insumos; de tal manera que la función de producción toma la forma $Y=A(t) f(K, L)$, donde $A(t)$ mide el efecto acumulado del cambio tecnológico en el tiempo. Al diferenciarse totalmente esta función respecto al tiempo $(t)$ y dividiéndose entre $Y$ es posible calcular a PTF como el diferencial entre el valor de lo elaborado y el costo del capital y del trabajo.

De manera análoga, al diferenciar la ecuación 1 totalmente respecto a $t$ y dividiendo entre $Y$, se tiene que:

$$
\frac{\dot{Y}}{Y}=\frac{\dot{A}}{A}+A\left[\frac{\partial f}{\partial e(w)} * \frac{e(w)}{Y}\right]+A\left[\frac{\partial f}{\partial L} * \frac{L}{Y}\right]
$$

donde el punto $(\cdot)$ sobre las literales indica cambios en el tiempo.

Por lo que $\alpha=\frac{\partial Y}{\partial e(w)} * \frac{e(w)}{Y}$, donde $\frac{\partial Y}{\partial e(w)}=A * \frac{\partial f}{\partial e(w)} ;$ y $\beta=\frac{\partial Y}{\partial L} * \frac{L}{Y}$, donde
$\frac{\partial Y}{\partial L}=A * \frac{\partial f}{\partial L}$.

Al sustituir $\alpha$ y $\beta$ en la ecuación 2 y simplificando la expresión en términos de tasas de crecimiento, es posible despejar a $L$ y llegar a lo siguiente:

$$
\frac{\dot{L}}{L}=\frac{\dot{Y}}{\beta Y}-\frac{\dot{A}}{\beta A}-\frac{\alpha e \dot{(w)}}{\beta e(w)}
$$

La ecuación 3 muestra que el producto $(Y)$ tiene una relación positiva con el empleo, mientras que $A$ y $w$ tienen un efecto negativo, tal como lo establece el enfoque de la NEK. Lo que resta es verificar esta relación teórica para la industria manufacturera mexicana mediante la estimación econométrica de los coeficientes.

En cuanto al análisis de la dimensión espacial, se argumenta que las aglomeraciones permiten el reforzamiento circular y acumulativo del crecimiento en regiones con alta concentración de empresas y revelan los mecanismos de convergencia-divergencia intra e inter-regional (Myrdal, 1957), lo cual se debe a ventajas como la expansión de los mercados comunes de bienes y de trabajo, la minimización de los costos de transporte de los insumos y de la producción, la generación de economías con ren- 
dimientos crecientes de escala y de externalidades tecnológicas, entre otras. En específico, al analizar las regiones como partes de un sistema se busca captar efectos interactivos entre ellas, pues éstas son más o menos interdependientes según la localización de su producción y consumo (Barro y Sala-i-Martín, 2004; Fujita et al., 1999; Sobrino, 2016).

En el mismo sentido, la NGE asume que la concentración da lugar a una relación positiva entre empleo, producción y salarios reales. En este enfoque teórico la producción de equilibrio de las empresas manufactureras en un lugar específico depende de su accesibilidad a los mercados y la competencia que enfrentan dichos mercados; por lo que la ubicación espacial ayuda a comprender la formación de aglomeraciones industriales en función de la proximidad geográfica (distancia) y las interacciones espaciales.

En la NGE se argumenta que las regiones deprimidas se caracterizan por una gran dispersión espacial de su producción y la prevalencia de sectores productivos intensivos en el uso de recursos naturales; en cambio, las regiones ricas presentan características exactamente contrarias (Fujita y Krugman, 2003). Para explicar estas diferencias, Krugman (1999) indica que existen fuerzas centrípetas o de atracción -como el tamaño del mercado, los eslabonamientos y la existencia de economías externas- que propician la concentración espacial de la producción, y fuerzas centrífugas o de dispersión -como la inmovilidad de los factores de producción, las rentas de la tierra y las des-economías externas- que conducen a menores niveles de desarrollo de las regiones.

\section{El empleo manufacturero en los estados mexicanos}

Los mercados laborales se reconfiguraron debido a los diversos choques experimentados por las regiones económicas del mundo; particularmente a partir de la crisis financiera de 2009 , -que en los años posteriores ha persistido de manera sistémica y estructural-, se evidenció que el modelo productivo vigente tiene limitaciones para absorber los excedentes laborales que ha generado. El aumento de la productividad a través del uso intensivo de la tecnología y la creciente dependencia hacia los flujos de inversión desviaron la atención hacia los movimientos especulativos de capital en el corto plazo y se descuidaron las estructuras productivas solventes (Merino, 2014).

El desempleo registrado en países de la Unión Europea y América Latina es reflejo de la creciente interdependencia de las economías y las políticas de flexibilización laboral que ampliaron la brecha entre los mercados primario y secundario de trabajo, que conlleva a redistribuciones 
espaciales vía migración poblacional y reubicación de unidades y actividades productivas (OIT, 2019).

Independientemente de la dotación de factores en las distintas regiones y países, la tendencia global ha conducido a una menor contribución del sector primario al producto y al empleo totales, en contraste con la mayor participación de las actividades terciarias; en cuanto al sector industrial, los cambios se han relacionado principalmente con el grado de desarrollo de los países y su capacidad de crecimiento (OIT, 2018).

La economía mexicana no ha sido ajena a esta dinámica global y -con el objetivo de insertarse convenientemente a ella, a través del tiempo- ha transitado por procesos de adaptación tendientes a incrementar la competitividad con el exterior, mejorar los sistemas productivos, captar mayores flujos internacionales de capital y crecer sostenidamente. Sin embargo, desde los ańos setenta se ha observado que el aparato productivo no genera los empleos que su crecimiento poblacional demanda (De Jesús Almonte, 2019).

La insuficiente generación de empleos se acentuó por la ocurrencia de eventos que interrumpieron las fases de crecimiento económico en México e impidieron consolidar las economías de aprendizaje; por lo tanto, el deterioro de la capacidad para crear nuevas fuentes de trabajo ha sido más que proporcional al retrasar el desarrollo de variables fundamentales como la inversión nacional y extranjera, la productividad y los salarios (Loría et al., 2019; Rodríguez y Castillo-Ponce, 2009).

$\mathrm{Al}$ sector industrial, en particular a la actividad manufacturera, se le ha atribuido efectos de arrastre que actúan como un "motor del crecimiento" de las economías estatales con mayor atraso. No obstante, el crecimiento industrial ha sido heterogéneo entre los subsectores que lo integran y en la tendencia de su distribución espacial, con la consecuente polarización de la producción, el ingreso y la capacidad de generación de empleo entre las regiones (Germán-Soto et al., 2010).

En congruencia con las tendencias globales, entre 1970 y 2015, el sector primario en México redujo sistemáticamente su contribución a la producción y al empleo totales, mientras que el sector terciario la incrementó progresivamente (cuadro 1).

$\mathrm{Al}$ interior del sector industrial se tuvieron comportamientos mixtos. La minería tendió a reducir su contribución a mediados de los años ochenta tras la caída de los precios del petróleo y de metales como el oro y la plata, así como por la política de diversificación de exportaciones. A causa de la campaña de electrificación del país y de fomento a los parques industriales, que se dio en los años setenta, el subsector de electricidad, gas y agua aumentó paulatinamente su contribución al producto total, aunque el incremento de su participación en el empleo fue mínimo dadas las inno- 


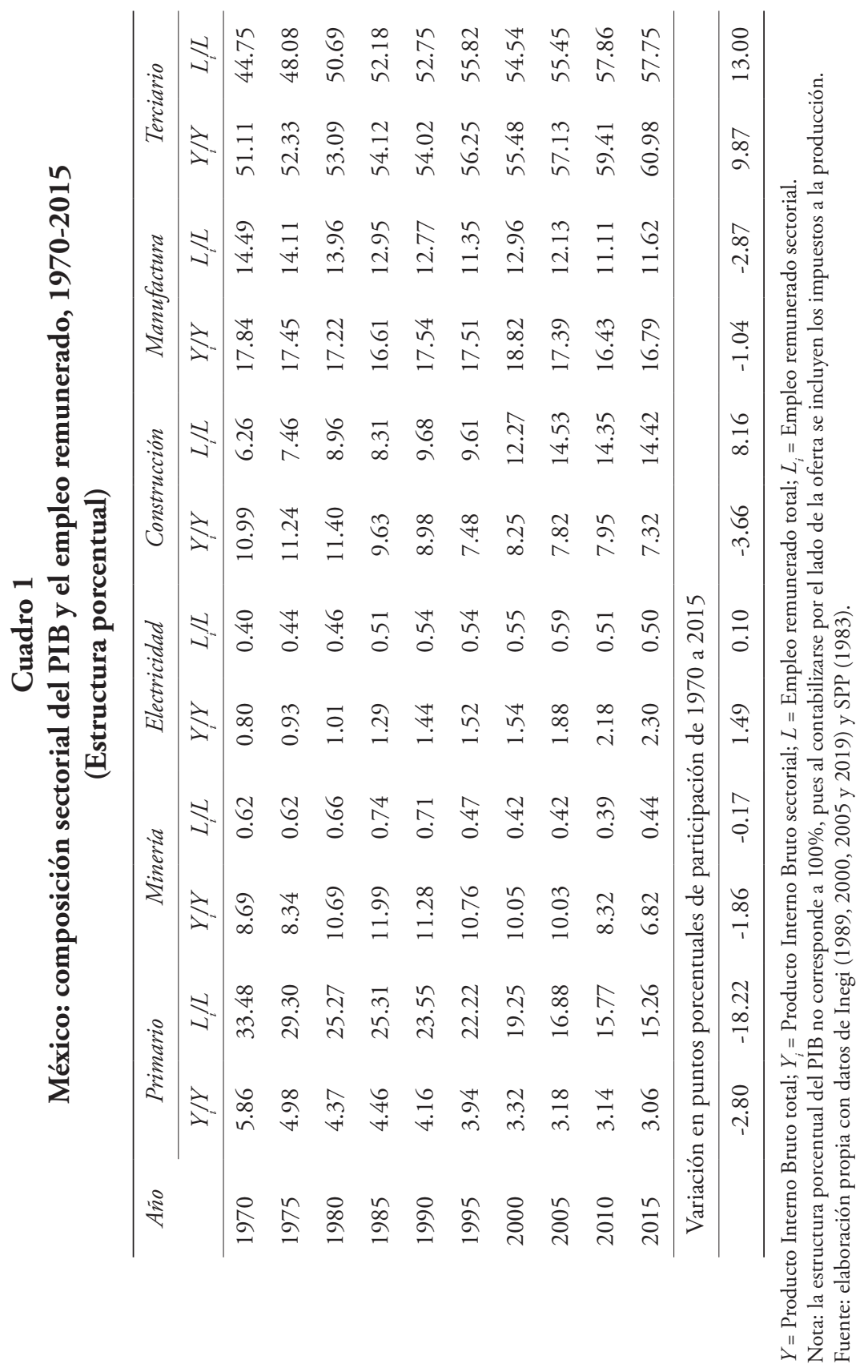


vaciones tecnológicas que experimenta esa actividad y a las pocas unidades productoras que la componen.

La construcción participó cada vez menos en la producción pero incrementó su contribución al empleo, lo cual supone una caída de su productividad laboral al considerarse que ha fungido como captadora de mano de obra expulsada de otras industrias o sectores. La manufactura mantuvo su contribución al producto en cerca de 17\%, pero disminuyó su participación en el empleo total; Pérez-Mendoza (2005) atribuye este comportamiento al aumento de las importaciones productivas, al uso de nuevas tecnologías, a la mayor productividad del factor capital experimentada en las décadas recientes y a la entrada de nuevos competidores al mercado global de manufacturas.

La evolución de la estructura porcentual del empleo manufacturero para cada estado (cuadro 2) se puede analizar a la luz de los siguientes puntos:

a) En los años setenta se acentuó la concentración de la manufactura en las zonas metropolitanas del Distrito Federal, ${ }^{6}$ Guadalajara y Monterrey; con el fin de alentar la descentralización de las empresas se ofrecieron atractivas condiciones para su reubicación y apoyos a las nuevas inversiones a través de un sistema diferenciado que beneficiaba a las ciudades medias que se deseaban promover; también se constituyeron fideicomisos para empresas manufactureras llamadas "tractoras," por considerarse que eran las que estructuraban las cadenas productivas, desarrollaban proveedores más eficientes y eran el ancla del crecimiento regional y de los agrupamientos industriales (Maldonado Carrasco, 2009). Los efectos de esta política de financiamiento fueron contraproducentes al estimular la concentración intrarregional y no a favor de reducir las desigualdades interregionales.

b) Se evidencia un particular rezago de la contribución a la ocupación manufacturera en las entidades situadas en la parte sur y sureste de México, cuyo perfil productivo está más orientado a actividades relacionadas con el sector primario y el de servicios y comercio. Lo cual se atribuye a su inherente dotación de recursos naturales y ubicación espacial.

c) Baja california Sur, Colima, Hidalgo, Morelos, Nayarit, Tlaxcala, Veracruz y Zacatecas destacan porque en el tiempo mantuvieron una baja contribución al empleo manufacturero o incluso la disminuyeron. ${ }^{7}$ Pese a que varias de estas entidades colindan con otras

\footnotetext{
${ }^{6}$ Aunque en 2017 cambió el nombre a Ciudad de México, por razones prácticas aquí se mantiene el nombre de esta entidad como fue registrada en los censos económicos.

${ }^{7}$ En los mapas del apéndice A se constata esta tendencia.
} 


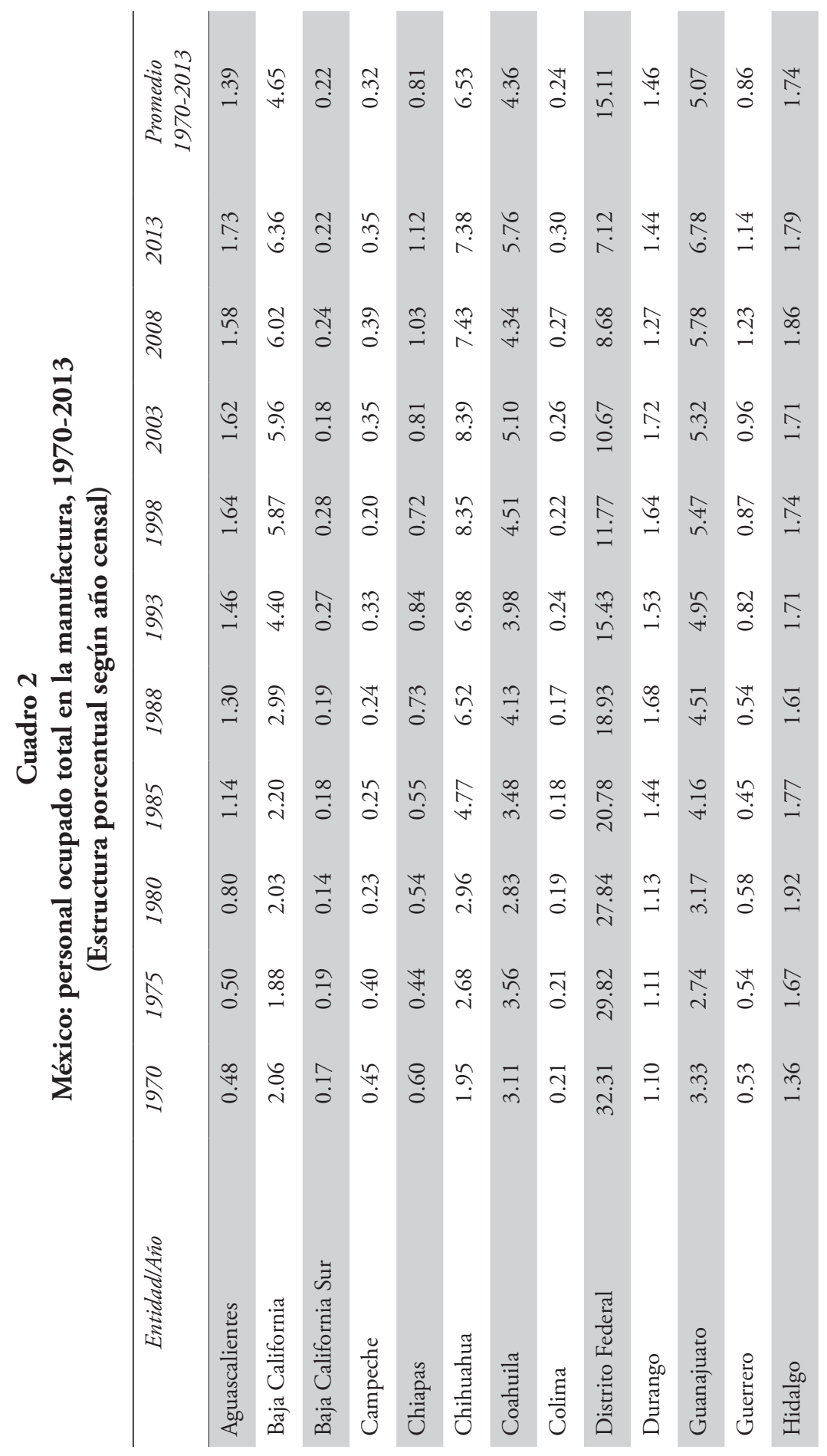




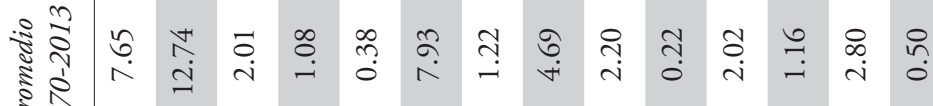

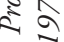

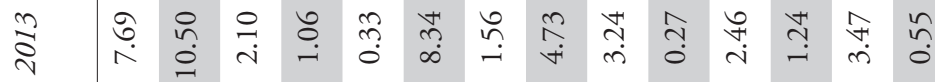

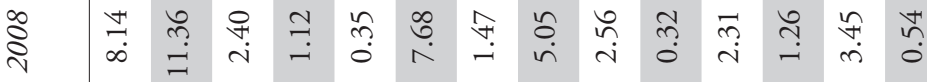

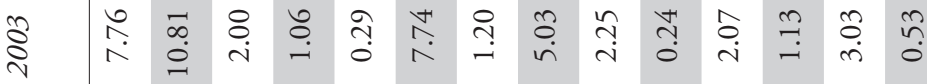

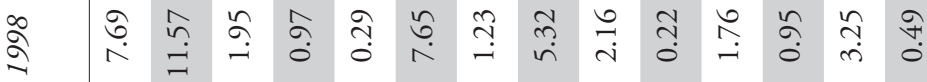

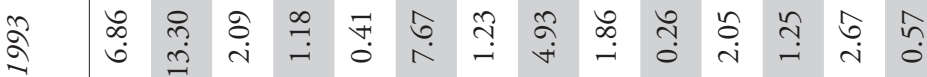

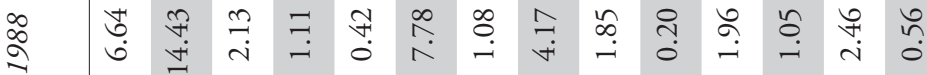

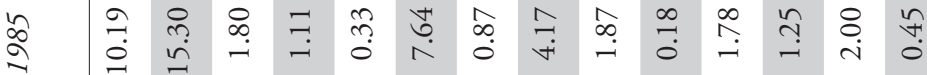

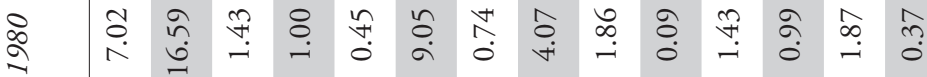

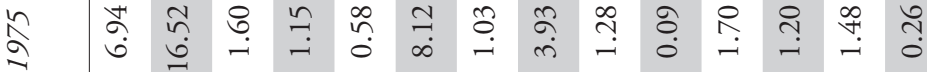

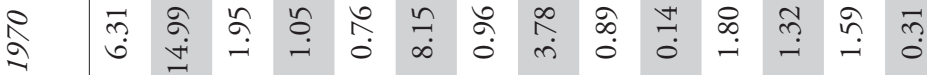




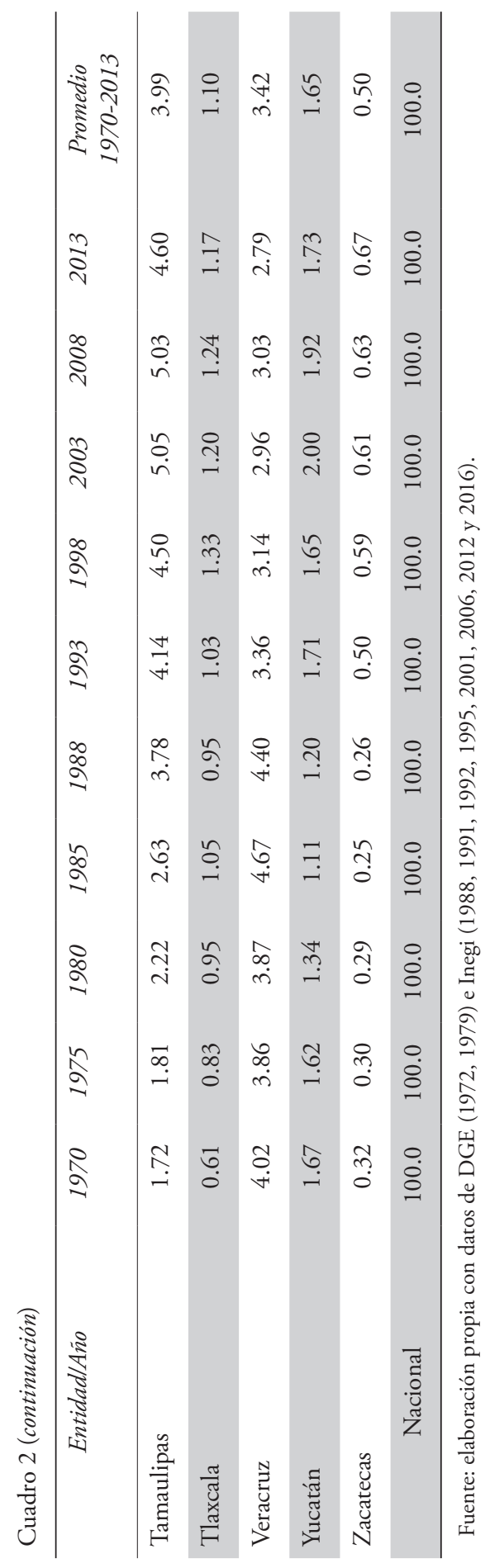


que tienen gran actividad manufacturera no han percibido los beneficios de dicha vecindad; las razones podrían radicar en su densidad poblacional, su infraestructura disponible e incluso el tamaño de su territorio, pues coincide que la mayoría son estados relativamente pequeños. ${ }^{8}$

En resumen, la política de descentralización industrial alcanzó parcialmente el objetivo de "re/ubicar" las empresas manufactureras; posteriormente, la transición de una economía cerrada hacia una abierta facilitó la aglomeración de esta industria en los estados de la frontera norte, centro-norte y centro-occidente, que terminaron por consolidarse tras la franca apertura comercial en 1994. El intento de industrializar a los estados del sur tuvo magros resultados.

De lo anterior se concluye que la distribución espacial de la actividad manufacturera ha implicado diferencias en la capacidad de generación de empleos de los estados, no sólo con relación a dicha industria sino también en otras e incluso sectores. Lo anterior es fundamental al considerar los efectos de arrastre derivados de la hipótesis kaldoriana, por lo que analizar la dependencia espacial ${ }^{9}$ permite entender los procesos que determinan al empleo en términos de las variables que intervienen.

\section{Metodología}

Se partió de un análisis de tipo exploratorio para verificar la existencia o no de interacciones espaciales entre los estados; posteriormente se realizó un análisis confirmatorio basado en la estimación de un modelo de panel de datos espaciales.

\subsection{Análisis exploratorio de datos espaciales (AEDE)}

Con la estimación del estadístico $I$ de Moran estimado de manera local y univariada se halló evidencia de autocorrelación espacial para la variable POT, aunque los resultados indican que sólo los periodos intercensales más recientes fueron estadísticamente significativos (cuadro 3). La dependencia espacial identificada a partir de 1993 puede explicarse por dos

\footnotetext{
${ }^{8}$ Para efectos del análisis econométrico, el problema relacionado con la inherente heterogeneidad en el tamańo y forma de los estados fue superado mediante el uso de centroides para medir la distancia entre las unidades espaciales y la especificación de una matriz de pesos espaciales con criterio Reina. Esto eliminó los posibles sesgos en la estimación de los atributos del fenómeno espacial bajo estudio.

${ }^{9}$ De acuerdo con Moreno Serrano y Vayá (2002: 86), "La dependencia o autocorrelación espacial aparece como consecuencia de la existencia de una relación funcional entre lo que ocurre en un punto determinado del espacio y lo que ocurre en otro lugar”.
} 


\section{Cuadro 3}

Autocorrelación espacial del empleo manufacturero por periodo intercensal

\begin{tabular}{lccc}
\hline \multirow{2}{*}{ Periodo } & \multicolumn{3}{c}{ POT } \\
\cline { 2 - 4 } & I de Moran & Valor $z$ & Valor \\
\hline $1970-1975$ & 0.120 & 1.208 & 0.118 \\
$1975-1980$ & -0.076 & -0.328 & 0.391 \\
$1980-1985$ & -0.119 & -0.678 & 0.245 \\
$1985-1988$ & 0.012 & 0.390 & 0.350 \\
$1988-1993$ & 0.100 & 1.111 & 0.137 \\
$1993-1998$ & 0.234 & 2.194 & 0.018 \\
$1998-2003$ & 0.169 & 1.836 & 0.028 \\
$2003-2008$ & 0.063 & 0.892 & 0.193 \\
$2008-2013$ & 0.213 & 1.892 & 0.034 \\
\hline
\end{tabular}

Fuente: elaboración propia con datos de DGE (1972, 1979) e Inegi $(1988,1991,1992,1995$, 2001, 2006, 2012 y 2016).

razones que favorecieron a la actividad manufacturera: la firma del Tratado de Libre Comercio de América del Norte, en 1992, y la modificación de la Ley de Inversión Extranjera Directa, en 1993.

A través del tiempo, las decisiones de instalación de nuevas empresas manufactureras y el traslado de las ya existentes tendieron a preferir a los estados fronterizos del norte, donde se aglomeran las entidades con alto crecimiento del empleo (mapa 1), mientras que en el sur las tasas de crecimiento son bajas.

Si bien esta conclusión no resulta novedosa, sí conlleva importantes implicaciones en términos estadísticos, al revelar interacciones espaciales entre los estados que pueden redundar en una preservación de las tendencias. En ese sentido, cuantificar los posibles efectos de retroalimentación mediante el análisis confirmatorio ayudaría a contrarrestar o revertir las desigualdades regionales.

\subsection{Metodología econométrica}

La evidencia de autocorrelación espacial reportada por el análisis exploratorio justifica tratar de manera conjunta a los datos del empleo manufac- 


\section{Mapa 1 \\ México: indicadores de asociación espacial local del empleo manufacturero (Periodos intercensales seleccionados)}
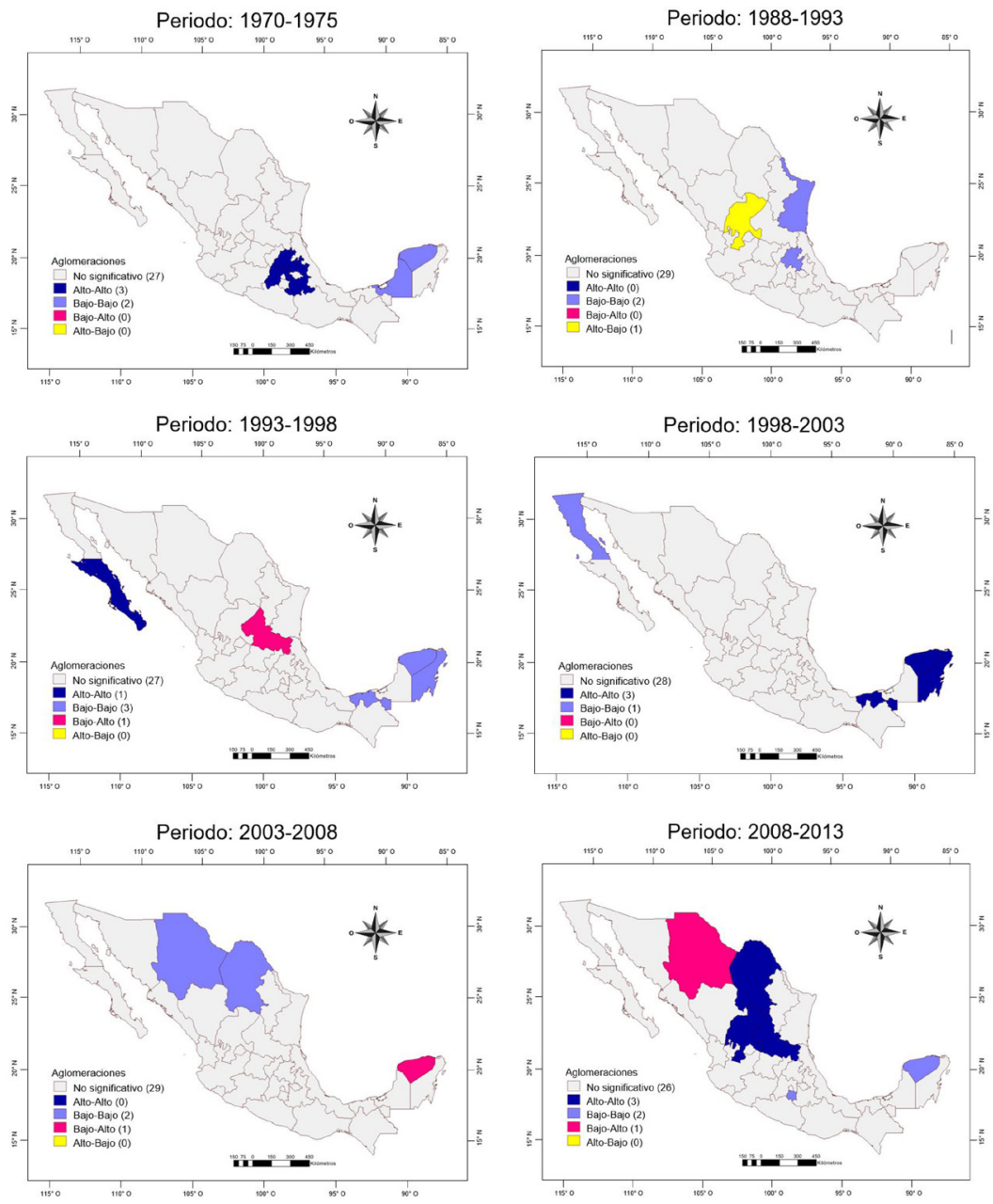

Fuente: elaboración propia con datos de DGE (1972, 1979) e Inegi $(1988,1991,1992,1995$, 2001, 2006, 2012 y 2016). 
turero y reproducirlos mediante un panel de datos espaciales. Al retomar la ecuación 3, la función del empleo se puede expresar como:

$$
P O T_{i t}=\alpha_{i t}+\beta_{1} P B T_{i t}+\beta_{2} R M_{i t}+\beta_{3} P T F_{i t}+u_{i t}
$$

Donde $i=1,2,3, \ldots, N$ unidades observadas, con $N$ igual a los 32 estados de México; $t=1,2, \ldots, T$ momentos en el tiempo, de modo que 1 corresponde al intervalo comprendido entre el primero y el segundo censo industrial registrado a partir de 1970; 2 corresponde al intervalo entre el segundo y el tercero, y sucesivamente hasta $T=9$.

Además, $\alpha$ representa un vector de interceptos de $K$ parámetros que contiene la función; $u$ es el término de error estocástico y POT, PBT, RM y PTF representan las TMAC del personal ocupado total, de la producción bruta total, de las remuneraciones medias reales pagadas al factor trabajo y de la productividad total de los factores, respectivamente. ${ }^{10}$

Se siguió el enfoque "de lo general a lo específico" propuesto por Elhorst $(2014)^{11}$ empezando por el modelo espacial de anidación general (GNS) ampliado con efectos específicos del periodo de tiempo, ${ }^{12}$ definido por la siguiente ecuación:

$$
Y_{t}=\rho W Y_{t}+\alpha l_{N}+X_{t} \beta+W X_{t} \theta+\xi_{t} l_{N}+u_{t}
$$

con $u_{t}=\lambda W u_{t}+\varepsilon_{t}$, y donde $Y_{t}$ es un vector $N \times 1$ de observaciones de la variable dependiente para cada estado $i$ en la muestra $(i=1,2, \ldots, 32)$ durante $T$ periodos de tiempo $(t=1,2, \ldots, 9) ; X_{t}$ denota una matriz $N \times K$ de variables explicativas exógenas. Además, $W$ es una matriz $N \times N$ de ponderaciones espaciales convencionales pre-especificada y estandarizada

${ }^{10}$ Con excepción de PTF, todas las variables son conocidas y directamente cuantificadas a partir de los datos definitivos de los censos industriales elaborados entre 1970 y 2013. Se utilizó el deflactor implícito del sector manufacturero (año base 2008). La estimación de PTF se basó en el residuo de Solow (1957), lo que evita la posible colinealidad entre las variables que se incluyen en la ecuación 4. El valor agregado censal bruto se tomó como una aproximación del producto $(Y)$; el total de remuneraciones y el acervo total de activos fijos se usaron como indicadores de la contribución del trabajo $(L)$ y del capital $(K)$, respectivamente (véase Inegi, 2013 y OECD, 2009). Los resultados fueron consistentes con los reportados por Díaz Bautista y Sáenz Castro (2002) y De León Arias (2008).

${ }^{11} \mathrm{La}$ consistencia de la especificación seleccionada se verificó de manera alternativa con la metodología "de lo específico a lo general" propuesta por Anselin et al. (1996), que parte del modelo agrupado estimado por mínimos cuadrados ordinarios (MCO) y a través del estadístico I de Moran se busca detectar autocorrelación espacial; en caso de hallarse, se identifica su tipo para luego pasar a especificaciones más elaboradas.

${ }^{12} \mathrm{Al}$ introducir el vector $\xi_{t}$, con un vector de parámetros $\gamma$, se incorpora una variable ficticia para cada unidad espacial y periodo de tiempo (excluyendo la intersección para evitar la colinealidad perfecta) que capta las heterogeneidades individuales y temporales, lo cual enriquece el análisis de los datos (Baltagi, 2005). 
por renglones, con entradas iguales a 1 si dos estados comparten un borde común y 0 en caso contrario (contigüidad tipo Reina).

Asimismo, $l_{N}$ es un vector $N \times 1$ de unos asociados con el parámetro constante $\alpha ; \mathrm{y}, \varepsilon=\left(\varepsilon_{1}, \ldots, \varepsilon_{N}\right)^{T}$ es un vector de términos de perturbación, donde $\varepsilon_{i}$ se asume que son términos de error independientes e idénticamente distribuidos (i.i.d.) para todo $i$ con media cero y varianza $\sigma^{2}$.

A $\rho$ se le denomina coeficiente autorregresivo espacial, $\lambda$ es el coeficiente de autocorrelación espacial de los errores $u_{i} \beta$ y $\theta$ representan cada uno un vector $K \times 1$ de parámetros fijos pero desconocidos que deben estimarse.

En el modelo GNS el efecto de la interacción endógena entre la variable dependiente queda expresado por $W Y$, la interacción exógena entre las variables independientes es cuantificado por $W X$ y la interacción entre las perturbaciones es verificable mediante el elemento $W u$.

Para examinar las diferencias entre los valores de la función logarítmica de verosimilitud, y elegir la especificación más adecuada, se aplicaron pruebas de razón de verosimilitud $(L R)$ convencionales que contrastan el valor de la función de probabilidad de registro bajo la hipótesis de que un modelo restringido en sus parámetros describe mejor los datos respecto a otro modelo no restringido.

Aunque representa una pérdida de grados de libertad, la estimación incluyó efectos fijos (fe) únicamente en el tiempo para controlar los fenómenos (choques) comunes que alteraron la trayectoria de las variables y generar inferencias condicionales de los datos que forman la muestra.

\section{Análisis espacial confirmatorio del empleo manufacturero}

La estimación del modelo agrupado por $\mathrm{MCO}$ aportó evidencia de autocorrelación espacial ( $I$ de Moran estadísticamente significativa) y con base en las pruebas $L M$ clásicas resultó indistinto optar por un modelo de error espacial o uno de rezago; sin embargo, las pruebas $F$ y Breusch-Pagan no aportaron evidencia concluyente para la selección de una especificación que incorpore efectos fijos o uno con efectos aleatorios (cuadro 4).

La significancia estadística (valor $p$ ) de los coeficientes fue la primera condición que debían cumplir para su posible selección; además, se realizó una evaluación comparativa de los modelos contrastando los valores de la razón de verosimilitud (LR), el $R^{2}$, la $\sigma^{2}$ y los criterios de información de Akaike (AIC) y bayesiano (BIC). Este proceso aportó elementos para seleccionar al GNS como el que mejor representa los datos de la TMAC intercensal del empleo en la manufactura. 


\section{Cuadro 4}

\section{Manufactura: estimación de POT mediante panel de datos espaciales}

\begin{tabular}{|c|c|c|}
\hline \multirow{2}{*}{$\begin{array}{l}\text { Variables } \\
\text { explicativas }\end{array}$} & Modelo 1 & Modelo 2 \\
\hline & $G N S \_f e$ & $M C O$ \\
\hline PBT & $0.446(0.000)$ & $0.458(0.000)$ \\
\hline RM & $-0.423(0.000)$ & $-0.335(0.000)$ \\
\hline PTF & $-0.082(0.000)$ & $-0.093(0.000)$ \\
\hline WPBT & $-0.244(0.002)$ & \\
\hline WRM & $0.272(0.016)$ & \\
\hline WPTF & $0.017(0.611)$ & \\
\hline Intercepto & & $1.184(0.000)$ \\
\hline$\rho$ & $0.506(0.000)$ & \\
\hline$\lambda$ & $-0.540(0.000)$ & \\
\hline$\sigma^{2}$ & 7.734 & 10.223 \\
\hline$R^{2}$ & 0.475 & 0.471 \\
\hline $\log L$ & -724.004 & -714.387 \\
\hline AIC & 1466.007 & 1490.774 \\
\hline BIC & 1498.974 & 1505.426 \\
\hline Prueba $\chi^{2} \mathrm{H}_{0}: \theta=0^{*}$ & $10.910(0.012)$ & n.a. \\
\hline Prueba de Hausman & n.a. & $0.230(0.973)$ \\
\hline Prueba $F$ & & $1.180(0.244)$ \\
\hline Breusch-Pagan $L M$ & & $0.330(0.283)$ \\
\hline I de Moran & & $2.836(0.005)$ \\
\hline LM-Err & & $6.951(0.008)$ \\
\hline LM-Lag & & $6.899(0.009)$ \\
\hline LM-Err ${ }_{\text {robusta }}$ & & $0.823(0.364)$ \\
\hline LM-Lag ${ }_{\text {robusta }}$ & & $0.772(0.380)$ \\
\hline
\end{tabular}

n.a. = Cálculo no aplicable.

* Prueba de significancia estadística conjunta de los coeficientes $\theta$.

Nota: Los valores $p$ se reportan entre paréntesis; el prefijo $W$ representa la matriz de pesos espaciales pre-especificada; el sufijo_fe indica efectos fijos.

Fuente: estimaciones propias. 
La ventaja de GNS se basa en que cuantifica la dependencia espacial ${ }^{13}$ mediante la observación de las interacciones exógenas, endógenas y entre los términos de error; también es posible medir los efectos directos e indirectos, así como los de retroalimentación.

El efecto autorregresivo, expresado por $\rho$, indica que manteniendo las demás variables sin cambios, $50 \%$ de las variaciones del empleo de una unidad específica son provocadas por las registradas en las unidades espaciales vecinas.

Las ideas expuestas referentes a la formación de mercados laborales comunes, la concentración de la producción y la ubicación cerca de las fuentes de materias primas y mercados de destino resaltan la importancia que tiene el hecho de que $\rho=0.506$ en el sentido de que la autocorrelación de los rezagos espaciales permite generar dinámicas virtuosas en este sector y favorece la aglomeración de unidades espaciales que comparten este atributo.

Llama la atención que el coeficiente de autocorrelación de los errores $(\lambda)$ tiene una magnitud similar al de los efectos autorregresivos $(\rho)$ pero con signo contrario; esto demuestra que el empleo de una entidad depende de los choques no observados y de los efectos de variables omitidos en el modelo pero provenientes de las entidades vecinas. El coeficiente $\lambda$ puede estar capturando las heterogeneidades generadas entre estados de alto crecimiento del empleo con otros de bajo crecimiento, lo que es congruente con lo reportado por Pérez-Mendoza (2005) al argumentar que este comportamiento indica que, particularmente, en la frontera norte se haya dado un crecimiento regional de la industria donde conviven un territorio desarrollado y otro subdesarrollado.

El efecto del coeficiente $\lambda=-0.54$ ayuda a comprender la bien intencionada pero fallida política de descentralización de la actividad manufacturera, pues las razones por las que no prosperó la creación de nuevas aglomeraciones se relacionan con heterogeneidades no consideradas inicialmente en dicha política gubernamental, tales como la insuficiente infraestructura, escasa fuerza de trabajo capacitada, lejanía de los merca-

\footnotetext{
${ }^{13}$ Por su naturaleza, las variables vinculadas al espacio presentan relaciones multidireccionales que pueden invalidar algunos supuestos de la econometría clásica y que se expresan en dos tipos de efectos. El primero es la dependencia espacial, que estadísticamente es similar a la autocorrelación serial, pero mientras ésta va en una sola dirección en el tiempo, la autocorrelación espacial vincula variables heterogéneas agrupadas en el espacio y, por tanto, plantea relaciones en las que cada observación posee distintas características en cuanto a tamaño, ubicación, distancias entre ellas, etc. Para contrastar la presencia de la dependencia espacial y expresar su estructura, en la econometría espacial se utilizan las matrices de pesos y los retardos espaciales, con los cuales es posible resolver el problema de la multidireccionalidad en la modelación. En cuanto al segundo efecto, la heterogeneidad espacial puede traer consigo resultados de estimación sesgados; sin embargo, puede manejarse mediante técnicas basadas en la econometría clásica. Pérez-Pineda (2006) resume y expone de manera didáctica las implicaciones de estos fenómenos de los datos y la forma de solucionarlos.
} 
dos, entre otros. Así, la mayoría de los estados de la región sur y otros como Durango y Nayarit son ejemplos del efecto negativo de $\lambda$; la presencia de estos fenómenos en dichas entidades dificultó la formación de aglomeraciones.

Los efectos directos espaciales expresados por el valor de los coeficientes $\beta$ presentan los signos sugeridos por la teoría, donde PBT es el principal determinante del empleo y los efectos negativos que generan RM son mayores a los de PTF. Esto muestra que el empleo es mucho más sensible a las variaciones de los salarios reales en comparación con los cambios que puedan producirse en términos de la productividad total factorial, sobre todo considerando la posible sustitución de fuerza de trabajo por capital derivada del aumento de la eficiencia marginal de este último factor.

La estimación de los efectos directos e indirectos permite inferir que la determinación del empleo manufacturero depende de manera muy equilibrada de las interacciones que se dan al interior de la misma unidad espacial $\mathrm{y}$ de las que provienen de unidades vecinas.

El hecho de que los efectos de derrame (indirectos) y los efectos directos tengan prácticamente el mismo peso revela que el sector es homogéneo en cuanto a sus mecanismos de transferencia de tecnología, la participación del capital y determinación de los salarios entre los estados que forman aglomeraciones. Este resultado confirma que en la industria manufacturera se desarrolla un reforzamiento circular y acumulativo que debería extenderse a otras regiones para promover la generación de empleo.

Los resultados del análisis econométrico indican la existencia de retroalimentación que explica por qué las variaciones en la producción de un estado particular tienen efectos cruciales en el empleo de la aglomeración entera, pues estos choques se transfieren a los estados vecinos y regresan a la unidad de origen con un efecto multiplicador de $10.7 \%$ sobre el valor del coeficiente de PBT, que es el principal determinante del empleo; mientras que las remuneraciones medias tienen un efecto similar en magnitud pero que actúa en sentido contrario, al igual que el de PTF (cuadro 5). La retroalimentación puede ser consecuencia de la información que tienen los agentes en cuanto al nivel de actividad (actual y futura) y los salarios pagados en otras entidades adyacentes, lo cual propicia la transferencia implícita de conocimiento y la movilidad laboral y de capitales. ${ }^{14}$

\footnotetext{
${ }^{14}$ Pese a no ser posible contrastar directamente los resultados con los de otros estudios, considerando la metodología econométrica empleada y las variables utilizadas para el análisis del empleo manufacturero, se observó que en general las magnitudes y signos de los distintos efectos de interacción espacial aquí reportados son compatibles con los hallazgos expuestos por De Jesús Almonte et al. (2020) y Mejía-Reyes y Torres-Preciado (2020).
} 


\begin{tabular}{lccc}
\multicolumn{5}{c}{ Cuadro 5 } \\
Manufactura: efectos directos e indirectos de las variables \\
explicativas de POT
\end{tabular}

* Refiere la contribución porcentual de los efectos de retroalimentación a la estimación de los coeficientes. El cálculo se basó en la diferencia entre el efecto directo y su respectivo coeficiente $\beta$.

Notas: Los valores $p$ son reportados entre paréntesis.

Fuente: estimaciones propias.

\section{Conclusiones}

El modelo GNS fue el que mejor representó los datos de empleo en el sector manufacturero, que se caracteriza por ser relativamente complejo, y su principal bondad es que justamente por su generalidad permite identificar todos los tipos de efectos derivados de las interacciones espaciales.

$\mathrm{Al}$ evaluar los efectos directos se corroboraron como válidas las hipótesis planteadas en esta investigación: PBT resultó ser el principal determinante del empleo con efectos positivos, en tanto que RM y PTF tienen efectos negativos pero diferenciados, en virtud de que las remuneraciones reales restringen mayormente el crecimiento del empleo.

De manera puntual, los principales hallazgos de la investigación fueron los siguientes:

a) El empleo en la manufactura mostró una tendencia de aglomeración que inició en los estados del centro, centro-occidente y, en menor medida, del norte de México, donde las correlaciones fueron entre entidades con mayor crecimiento (alto-alto); hasta mediados de la década de los ochenta estas correlaciones se identificaron en algunos estados del sur del país, aunque ya para los años noventa fueron pocas y de tipo bajo-bajo. A partir de la completa apertura comercial es marcada la formación de aglomeraciones en los estados de la frontera norte con correlaciones alto-alto. 
Estos comportamientos exhiben una discontinuidad en el proceso de industrialización de las entidades y se asocian con fenómenos como las crisis recurrentes experimentadas en los años ochenta y las acciones del gobierno federal orientadas, primero, a la descentralización industrial y, después, al fomento de las exportaciones.

b) Se corroboró que a nivel espacial existe una relación negativa empleo-salarios reales $(-0.471)$ y empleo-productividad (-0.089). Este hallazgo es congruente con lo reportado por Lechuga Montenegro y Varela Orozco (2001), quienes entre 1990 y 1998, con datos agregados de la manufactura, refirieron una relación inversa entre productividad y empleo en la que por cada punto porcentual que incrementa esta variable explicativa el empleo se reduce en $0.53 \%$.

c) La no significancia estadística de los efectos indirectos de la PTF indica que los cambios tecnológicos a nivel regional no son relevantes en las variaciones del empleo, pues si bien en el sector existe una continua renovación de maquinaria y equipo, así como uso de nuevas tecnologías de información y comunicaciones, el mayor crecimiento del empleo se registra en las actividades relacionadas con la industria maquiladora de exportación, en la que los salarios son bajos y se realizan únicamente tareas de ensamblado (Escobar Méndez, 2011; Mendoza Cota, 2009).

d) Las tres variables explicativas registran tasas de retroalimentación de magnitud similar, estableciéndose que al interior de las aglomeraciones se presentan dinámicas virtuosas de producción, transferencia de tecnología (del extranjero, de otros sectores industriales y entre las ramas de actividad manufactureras) y un mercado laboral más homogéneo en términos de capacitación, niveles salariales y localización espacial.

De acuerdo con los resultados, la tendencia de escaso crecimiento del empleo y su concentración espacial parece irreversible para los próximos años, a menos que se entre en una nueva fase de crecimiento económico sostenido y se implementen reformas laborales encaminadas a elevar la elasticidad, producto del empleo.

Asimismo, el esfuerzo por elevar la ocupación deberá apoyarse en el fomento de la contratación formal y en trasladar a los trabajadores los beneficios del incremento de la productividad laboral a través de niveles salariales que compensen la pérdida acumulada del poder adquisitivo. Esto es viable al considerar el efecto "mercado interno", postulado por la NGE, el cual asume que el incremento en el ingreso del mercado interno en una ubicación específica provocará un aumento en el empleo y la producción manufacturera de esa ubicación, y parte de la producción 
adicional podrá exportarse a otros lugares accesibles; así, el ingreso interno y el externo influirán positivamente en el empleo manufacturero local (Fujita et al., 1999). Un aumento en la demanda efectiva desplazaría la curva de demanda laboral hacia arriba, generando un mayor nivel de empleo dado el salario de eficiencia y la curva de oferta de trabajo.

Debido a que la manufactura se caracteriza por una fuerte interdependencia espacial, la creación/localización de parques industriales debe considerar este factor decisivo, sobre todo en estados donde la actividad manufacturera es incipiente y puede impulsarse su crecimiento generando dinámicas virtuosas basadas en los efectos de retroalimentación (supuesto de reforzamiento circular y acumulativo).

Este acercamiento al análisis espacial del empleo en la industria manufacturera cubre el objetivo de identificar los principales factores que han facilitado la formación de aglomeraciones en ciertas regiones y dificultado su desarrollo en otras; asimismo, cumple la expectativa de evaluar los efectos espaciales que han tenido los choques económicos y los mecanismos que incentivan o inhiben la creación de empleos. Sin embargo, queda pendiente un análisis de lo que ocurre al interior de las regiones en términos de las interacciones entre las unidades espaciales que las conforman, incluso a nivel de municipios o de ciudades principales, y aprovechar la desagregación de los datos por ramas de actividad para vislumbrar los procesos subyacentes a la generación de empleo.

Debido a que los datos utilizados para el análisis fueron de tipo censal, una de las principales limitaciones del trabajo fue la imposibilidad de extender el estudio hasta el 2019, al no contar con resultados definitivos del más reciente censo económico; hacerlo habría permitido abordar los posibles efectos espaciales que ha tenido la crisis financiera de 2009 y que persisten hasta años recientes. 


\section{Apéndice A}

\section{México: TMAC del empleo manufacturero por cuantiles y periodos seleccionados}
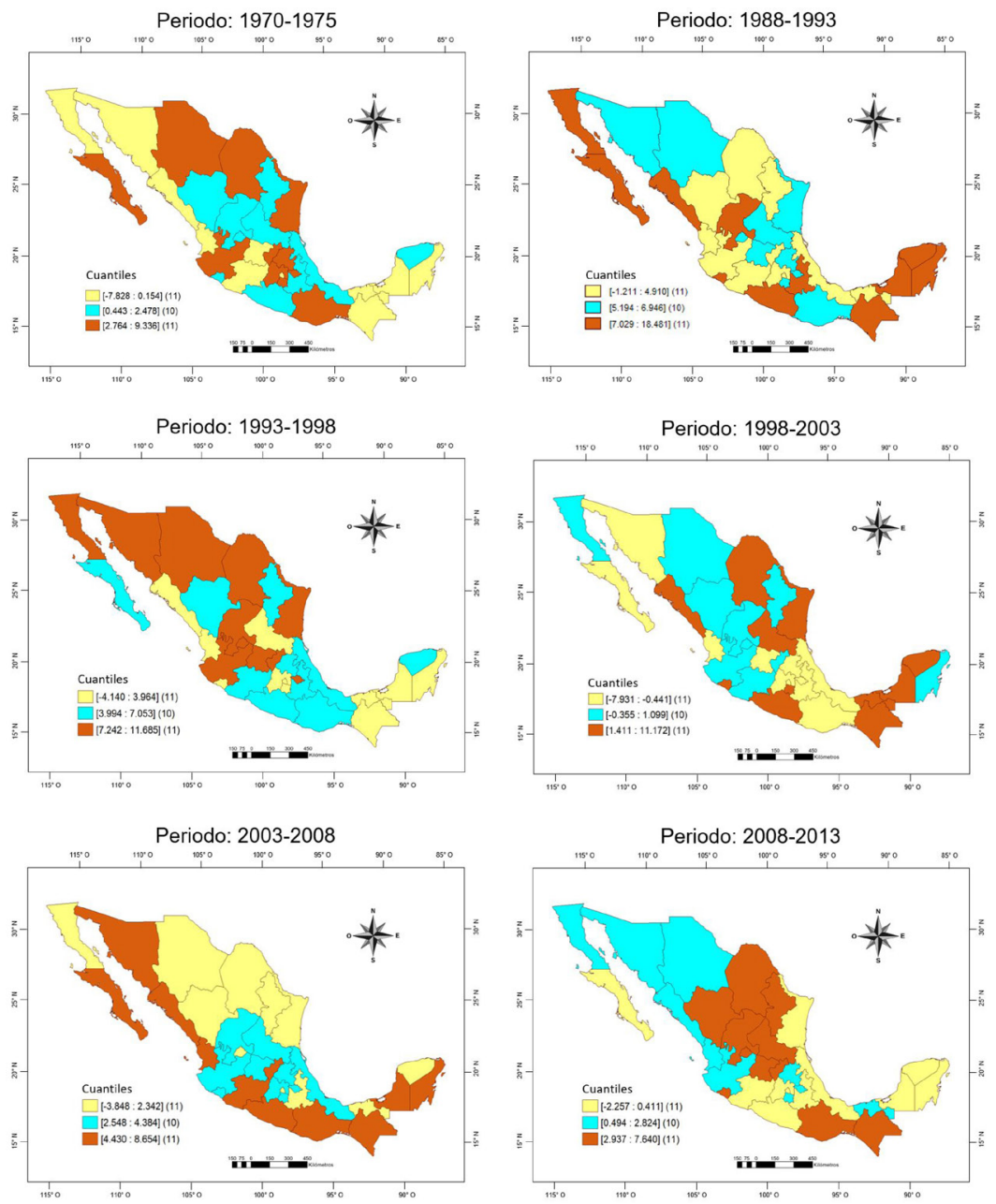

Fuente: elaboración propia con datos de DGE (1972, 1979) e Inegi (1988, 1991, 1992, 1995, 2001, 2006, 2012 y 2016). 


\section{Fuentes consultadas}

Anselin, Luc; Bera, Anil Kumar; Florax, Raymond y Yoon, Mann (1996), "Simple diagnostic tests for spatial dependence", Regional Science and Urban Economics, 26 (1), Ámsterdam, Elsevier, pp. 77-104.

Asuad Sanén, Normand y Quintana Romero, Luis (2010), "Crecimiento económico, convergencia y concentración económica espacial en las entidades federativas de México 1970-2008", Investigaciones Regionales, núm. 18, Alcalá de Henares, Asociación Española de Ciencia Regional, pp. 83-106.

Baltagi, Badi (2005), Econometric analysis of panel data, Chichester, John Wiley \& Sons Ltd.

Barro, Robert y Sala-i-Martín, Xavier (2004), Economic growth, London, The MIT Press.

De Jesús Almonte, Leobardo (2019), Lento crecimiento y empleo manufacturero en México. Un análisis de endogeneidad territorial, Toluca, Ediciones EON-Universidad Autónoma del Estado de México.

De Jesús Almonte, Leobardo; Andrés Rosales, Roldán y Carbajal Suárez, Yolanda (2020), "Spatial analysis of manufacturing employment in Mexico, 1984-2013", Revista Desarrollo y Sociedad, núm. 84, Bogotá, Universidad de los Andes, pp. 91-129.

De León Arias, Adrián (2008), "Cambio regional del empleo y productividad manufacturera en México. El caso de la frontera norte y las grandes ciudades: 1970-2004", Frontera Norte, 20 (40), Tijuana, El Colegio de la Frontera Norte, pp. 79-103.

DGE (Dirección General de Estadística) (1979), X Censo Industrial, 1976. Resumen general, Ciudad de México, Secretaría de Programación y Presupuesto-Dirección General de Estadística.

DGE (Dirección General de Estadística) (1972), IX Censo Industrial, 1971. Resumen general, Ciudad de México, Secretaría de Industria y Comercio-Dirección General de Estadística.

Díaz Bautista, Alejandro y Sáenz Castro, Jorge Enrique (2002), "Productividad total factorial y el crecimiento económico en México", 
Economía y Desarrollo, 1 (1), Bogotá, Fundación Universidad Autónoma de Colombia, pp. 105-180.

Elhorst, Jean Paul (2014), Spatial econometrics from cross-sectional data to spatial panels, Berlín, Springer.

Escobar Méndez, Aracely (2011), "Determinantes del empleo en la industria manufacturera en México", Papeles de Población, 17 (67), Toluca, Universidad Autónoma del Estado de México, pp. 251-276.

Fujita, Masahisa y Krugman, Paul (2003), “The new economic geography: past, present and the future", Papers in Regional Science, 83 (1), Hoboken, Wiley-Blackwell, pp. 139-164.

Fujita, Masahisa; Krugman, Paul y Venables, Anthony (1999), The spatial economy. Cities, regions, and international trade, Cambridge, The MIT Press.

Garrocho, Carlos (2012), Estructura funcional de la red de ciudades de México, Zinacantepec, El Colegio Mexiquense-Consejo Nacional de Población-Fondo de Población de las Naciones Unidas.

Germán-Soto, Vicente; Escobedo Sagaz, José Luis y Flores Gallegos, Luis (2010), "Convergencia y divergencia en el sector industrial de los estados mexicanos: un análisis espacial no paramétrico", Equilibrio Económico, 6 (2), Saltillo, Universidad Autónoma de Coahuila, pp. 161-186.

Gordon, Robert James (1990), "What is New-Keynesian Economics?", Journal of Economic Literature, 28 (3), Nashville, American Economic Association, pp. 1115-1171.

Inegi (Instituto Nacional de Estadística y Geografía) (2019), Sistema de Cuentas Nacionales de México. Cuentas de bienes y servicios 2003-2018, Aguascalientes, Inegi. <https:/www.inegi.org.mx/ $\mathrm{app} / \mathrm{tmp} /$ tabuladoscn/default.html?tema $=\mathrm{CBS}>, 5$ de septiembre de 2019.

Inegi (Instituto Nacional de Estadística y Geografía) (2016), XVII Censo Industrial. Censos Económicos 2014, Aguascalientes, Inegi. 
Inegi (Instituto Nacional de Estadística y Geografía) (2013), Sistema de Cuentas Nacionales de México. Productividad total de los factores 1990-2011, Aguascalientes, Inegi.

Inegi (Instituto Nacional de Estadística y Geografía) (2012), XVI Censo Industrial. Censos Económicos 2009, Aguascalientes, Inegi.

Inegi (Instituto Nacional de Estadística, Geografía e Informática) (2006), XV Censo Industrial. Censos Económicos 2004, Aguascalientes, Inegi.

Inegi (Instituto Nacional de Estadística, Geografía e Informática) (2005), Sistema de Cuentas Nacionales de México. Cuentas de bienes y servicios 1999-2004, Tomo I, Aguascalientes, Inegi.

Inegi (Instituto Nacional de Estadística, Geografía e Informática) (2001), XIV Censo Industrial. Censos Económicos 1999, Aguascalientes, Inegi.

Inegi (Instituto Nacional de Estadística, Geografía e Informática) (2000), Sistema de Cuentas Nacionales de México. Cuentas de Bienes y Servicios 1988-1999, Tomo I, Aguascalientes, Inegi.

Inegi (Instituto Nacional de Estadística, Geografía e Informática) (1995), XIV Censo Industrial. Censos Económicos 1994, Aguascalientes, Inegi.

Inegi (Instituto Nacional de Estadística, Geografía e Informática) (1992), XIII Censo Industrial. Censos Económicos 1989, Aguascalientes, Inegi.

Inegi (Instituto Nacional de Estadística, Geografía e Informática) (1991), XII Censo Industrial. Censos Económicos 1986, Aguascalientes, Inegi.

Inegi (Instituto Nacional de Estadística, Geografía e Informática) (1989), Sistema de Cuentas Nacionales de México, 1981-1987. Resumen General, Tomo I, Aguascalientes, Inegi.

Inegi (Instituto Nacional de Estadística, Geografía e Informática) (1988), XI Censo Industrial. Censos Económicos 1981, Aguascalientes, Instituto Nacional de Estadística, Geografía e Informática. 
Krugman, Paul (1999), "The role of geography in development", en Boris Pleskovic y Joseph Stiglitz (eds.), Annual World Bank Conference in Development Economics 1998, Washington, World Bank, pp. 89-107.

Lechuga Montenegro, Jesús y Varela Orozco, Mauricio (2001), “Empleo manufacturero en México, 1990-1998”, Análisis Económico, 16 (33), Ciudad de México, Universidad Autónoma Metropolitana unidad Azcapotzalco, pp. 215-234.

Loría, Eduardo; Moreno Brid, Juan Carlos; Salas, Emmanuel y Sánchez Juárez, Isaac (2019), "Explicación kaldoriana del bajo crecimiento económico en México", Problemas del Desarrollo. Revista Latinoamericana de Economía, 50 (196), Ciudad de México, Universidad Nacional Autónoma de México, pp. 3-26.

Maldonado Carrasco, Ana (2009), "Parques industriales de México: dos perspectivas de desarrollo", Comercio Exterior, 59 (1), Ciudad de México, Banco Nacional de Comercio Exterior, pp. 60-76.

Mankiw, Gregory y Romer, David (eds.) (1991), New Keynesian Economics, vol. I y II, Cambridge, The MIT Press.

Mejía-Reyes, Pablo y Torres-Preciado, Víctor Hugo (2020), “Determinants of manufacturing employment in the Mexican states, 20042017”, Regional Science Policy and Practice, 12 (2), Chichester, John Wiley \& Sons Ltd., pp. 303-318, doi:https://doi. org/10.1111/rsp3.12245

Mendoza Cota, Jorge Eduardo (2009), "Las exportaciones de China y los determinantes locales del empleo en las maquiladoras de la frontera norte de México", Región y Sociedad, 21 (44), Hermosillo, El Colegio de Sonora, pp. 145-169.

Merino, Amparo (2014), "Los efectos de la crisis en el empleo. Integración económica, Estado de bienestar y medidas de fomento del (des)empleo", IUS, 8 (33), Puebla, Instituto de Ciencias Jurídicas, pp. 59-76.

Moreno Serrano, Rosina y Vayá, Esther (2002), "Econometría espacial: nuevas técnicas para el análisis regional. Una aplicación a las regiones europeas", Investigaciones Regionales, núm. 1, Madrid, Asociación Española de Ciencia Regional, pp. 83-106. 
Myrdal, Gunnar (1957), Economic theory and underdeveloped regions, Londres, Duckworth.

Ocegueda Hernández, Juan Manuel (2015), “Divergencias de tasas de crecimiento entre las economías estatales de México, 19932010", Región y Sociedad, 27 (64), Hermosillo, El Colegio de Sonora, pp. 139-182.

OECD (Organization for Economic Co-operation and Development) (2009), Measuring capital. OECD Manual, París, OECD Publishing.

OIT (Organización Internacional del Trabajo) (2019), Perspectivas sociales y del empleo en el mundo: tendencias 2019, Ginebra, Oficina Internacional del Trabajo.

OIT (Organización Internacional del Trabajo) (2018), Perspectivas sociales y del empleo en el mundo: tendencias 2018, Ginebra, Oficina Internacional del Trabajo.

Pérez-Mendoza, Arturo (2005), "Liberalización comercial y la creación y destrucción de empleo", Estudios Económicos, 20 (1), Ciudad de México, El Colegio de México, pp. 79-108.

Pérez-Pineda, Jorge (2006), "Econometría espacial y ciencia regional", Investigación Económica, 65 (258), Ciudad de México, Universidad Nacional Autónoma de México, pp. 129-160.

Rodríguez Benavides, Domingo; Mendoza González, Miguel Ángel y Venegas Martínez, Francisco (2016), “¿Realmente existe convergencia regional en México? Un modelo de datos-panel TAR no lineal", Economía, Sociedad y Territorio, 16 (50), Zinacantepec, El Colegio Mexiquense, pp. 197-227.

Rodríguez, María de Lourdes y Castillo-Ponce, Ramón (2009), “Empleo, productividad y salarios en México: un análisis de corto y de largo plazo para el sector manufacturero", EconoQuantum, 5 (2), Zapopan, Universidad de Guadalajara, pp. 7-21.

Schlicht, Ekkehart (2016), "Efficiency wages: variants and implications", IZA World of Labor, núm. 275, Bonn, Institute of Labor Economics, pp. 1-10, <https://wol.iza.org/articles/efficiency-wagesvariants-and-implications $>, 10$ de marzo de 2020. 
Sobrino, Jaime (2016), "Localización industrial y concentración geográfica en México”, Estudios Demográficos y Urbanos, 31 (1), Ciudad de México, El Colegio de México, pp. 9-56.

Solow, Robert (1957), “Technical change and the aggregate production function", The Review of Economics and Statistics, 39 (3), Cambridge, The MIT Press, pp. 312-320.

SPP (Secretaría de Programación y Presupuesto) (1983), Principales variables macroeconómicas. Periodo 1970-1982, Ciudad de México, SPP-Inegi.

World Bank (2009), World Development Report 2009: Reshaping Economic Geography, Washington, World Bank.

Recibido: 26 de noviembre de 2019. Reenviado: 24 de febrero de 2020. Aceptado: 17 de abril de 2020 .

Luis Brito Cruz. Maestro en economía financiera por la Facultad de Economía de la Universidad Autónoma del Estado de México, actualmente es doctorante en Ciencias Económico Administrativas por la misma universidad. Sus líneas de investigación son empleo sectorial, inversión extranjera directa y productividad. Entre sus más recientes publicaciones se encuentran, como autor: "El empleo y la desocupación: un análisis desde los enfoques teóricos keynesianos", en Patricia Mercado Salgado y Daniel Arturo Cernas Ortiz (coords.), Aportaciones a los Estudios Económico-Administrativos. Reflexiones teóricas y evidencias empíricas, Toluca, Universidad Autónoma del Estado de México, pp. 285-307 (2016); como coautor, "Censos económicos 2009 (cifras preliminares): un breve análisis del rumbo de las Mipymes en México", Economía Actual, 3 (2), Toluca, Universidad Autónoma del Estado de México, pp. 34-37 (2010), y como coautor, "El impacto de la inversión extranjera directa en el empleo sectorial en México: un análisis prospectivo", Análisis Económico, 20 (44), Ciudad de México, Universidad Autónoma Metropolitana unidad Azcapotzalco, pp. 5-34 (2005).

Pablo Mejía Reyes. Doctor en Economía por la Universidad de Manchester, actualmente es investigador del Centro de Investigación en Ciencias Económicas de la Facultad de Economía de la Universidad Autónoma del Estado de México y miembro del Sistema Nacional de 
Investigadores, nivel 3. Sus líneas de investigación son: fluctuaciones cíclicas, integración económica internacional, macroeconomía subnacional aplicada y econometría aplicada. Entre sus publicaciones más recientes se encuentran, en coautoria: "Determinants of manufacturing employment in the Mexican states, 2004-2017", Regional Science Policy \& Practice, 12 (2), Chichester, John Wiley \& Sons Ltd., pp. 303-318 (2020); "Mexican states' business cycles co-movement over the period 2000-2014. A panel data model estimation", Growth and Change, 50 (4), Chichester, John Wiley \& Sons Ltd., pp. 1532-1567 (2019) y "Ciclos económicos y gasto público: un análisis de cambio estructural para la región centro de México, 1980-2015”, Paradigma Económico, 11 (2), Toluca, Universidad Autónoma del Estado de México, pp. 5-38 (2019). 